01

\title{
Параметризованный эффективный потенциал для возбужденных состояний и его применение к расчету дипольных моментов перехода
}

\author{
(C) В.Н. Глушков, С.И. Фесенко \\ Днепровский национальный университет им. О. Гончара, \\ 49000 Днепр, Украина \\ e-mail: v_n_glushkov@yahoo.com
}

Поступила в редакцию 05.11.2019 г.

В окончательной редакции 05.01.2020 г.

Принята к публикации 09.01.2020 г.

\begin{abstract}
Метод параметризованного эффективного потенциала, предложенный ранее для основного состояния, развит для возбужденных состояний с симметрией основного. Обсуждены различные приближения для определения обменно-корреляционных потенциалов, $V_{x c}$. В частности, на основе требования симметрии $V_{x c}$ представлен один из рецептов построения нового класса $V_{x c}$, который является функционалом внешнего потенциала (взаимодействия электрон-ядро) в отличие от традиционных подходов, где $V_{x c}$ явно или неявно зависят от электронной плотности. Возможности метода исследованы на примере расчета электронных дипольных моментов перехода молекулы НеН с однодетерминантной волновой функцией. Показано, что предложенный метод расчета дипольных моментов перехода можно рассматривать как компромисс между точностью и вычислительными усилиями.
\end{abstract}

Ключевые слова: параметризованный эффективный потенциал, дипольные моменты перехода, возбужденные состояния.

DOI: $10.21883 /$ OS.2020.04.49193.299-19

\section{Введение}

C момента публикации основополагающих работ Hohenberg-Kohn-Sham [1,2] теория функционала плотности (ТФП или DFT) стала ведущим и популярным инструментом в изучении электронной структуры атомов, молекул и твердых тел. Некоторые исторические аспекты, основные концепции и оценку работоспособности растущего числа приближенных обменнокорреляционных функционалов $V_{x c}(\mathbf{r})$ можно найти в превосходных обзорах [3-5]. Известно, что успех ТФП во многом определяется выбором $V_{x c}(\mathbf{r})$. К сожалению, сама ТФП не дает практических рецептов их построения. В ходе развития ТФП были предложено большое число $V_{x c}(\mathbf{r})$ функционалов, которые различаются точностью аппроксимаций (см., например, [5-7]). В стандартной теории Kohn-Sham потенциалы $V_{x c}(\mathbf{r})$ явно или неявно зависят от электронной плотности $\rho(\mathbf{r})$, т.е. $V_{x c}(\mathbf{r})=V_{x c}[\rho(\mathbf{r})]$. Было показано, что плотность, не имеющая симметрии внешнего потенциала, дает потенциалы KS

$$
V_{\mathrm{KS}}(\mathbf{r})=V_{\mathrm{ext}}(\mathbf{r} r)+V_{H}(\mathbf{r})+V_{x c}(\mathbf{r})
$$

которые не могут воспроизвести корректную плотность, если не наложены дополнительные ограничения [8]. В уравнении (1) $V_{\text {ext }}(\mathbf{r})$ и $V_{H}(\mathbf{r})$ - соответственно внешний и кулоновский потенциалы. Другими словами, $V_{\mathrm{KS}}(\mathbf{r})$ не обладает симметрией внешнего потенциала из-за асимметрии электронной плотности. Подробное обсуждение проблемы асимметрии в ТФП и ее последствий можно найти в работах [8-12].

В недавней работе A.K. Theophilou [12] доказал теорему, которая утверждает, что в атомах, молекулах и твердых телах только набор сферических частей плотности вокруг каждого ядра однозначно определяет внешний потенциал. Другими словами (см. теорему 2 в [12]), полный потенциал $V_{\mathrm{KS}}(\mathbf{r})$ представляет собой сумму потенциалов, каждый из которых сферически симметричен относительно вращения вокруг соответствующих ядер. Альтернативное доказательство этой теоремы было дано A. Nagy [13]. Эта теорема дает подсказку для построения нового класса обменно-корреляционных функционалов энергии и соответствующего потенциала $V_{\mathrm{KS}}(\mathbf{r})$ на их основе.

Одна из возможных форм эффективного потенциала $V_{\mathrm{ef}}(\mathbf{r})$, который удовлетворяет условиям теоремы и играет роль KS потенциала в атомах и молекулах, была предложена в $[14,15]$. В духе соображений, изложенных выше, этот потенциал был выражен в виде прямого отображения внешнего потенциала, а именно

$$
\begin{aligned}
V_{\mathrm{ef}}(\mathbf{r})= & -\Sigma_{k} \frac{z_{k}}{\left|\mathbf{r}-\mathbf{R}_{k}\right|}-\frac{N-1}{Z} C \Sigma_{k} Z_{k} \\
& \times \frac{1-\exp \left(-d_{k}\left|\mathbf{r}-\mathbf{R}_{k}\right|\right)}{\left|\mathbf{r}-\mathbf{R}_{k}\right|}
\end{aligned}
$$

где $Z=\Sigma_{k} Z_{k}$ и суммирование проводится по ядрам с радиусами-векторами $\mathbf{R}_{k}$ и зарядом $Z_{k}, N$ - число электронов. Вариационные параметры $C$ и $d_{k}$ определяются 
минимизацией функционала энергии $E(\Phi)=\langle\Phi|H| \Phi\rangle$, где $\Phi$ - это $N$-частичный детерминант Слэтера, который описывает систему невзаимодействующих частиц. В такой имплементации $V_{\mathrm{ef}}(\mathbf{r})$ близок к потенциалу метода оптимизированного эффективного потенциала [16-18]. Эффективный потенциал в виде (2) оказался полезным при определении энергии систем с замкнутыми и открытыми оболочками как для основного, так и возбужденного состояний [19-21] в приближении только обменного потенциала, т. е. когда в представлении

$$
V_{x c}(\mathbf{r})=V_{x}(\mathbf{r})+V_{c}(\mathbf{r})
$$

ограничиваются только обменным потенциалом $V_{x}(\mathbf{r})$. Возможности учета статической части корреляционного потенциала $V_{c}(\mathbf{r})$ были рассмотрены в работе [22]. В нашей ранней работе [23] такой метод определения энергии был назван как метод параметризованного эффективного потенциала (ПЭП).

В работах $[24,25]$ нами были представлены результаты вычислений электронных дипольных моментов перехода (ДМП) в молекуле НеН, полученные с однодетерминантной волновой функцией в рамках приближения Хартри-Фока (ХФ). Стоит отметить, что, насколько нам известно, в литературе отсутствуют расчеты ДМП в однодетерминантном приближении для переходов между состояниями одинаковой пространственной симметрии, хотя для состояний разной пространственной и спиновой симметрии такие попытки существуют, начиная с работы [26]. Целью данной работы является исследование возможности применения метода ПЭП для расчета ДМП на примере молекулы НеН и сравнение соответствующих результатов с результатами в приближении ХФ и высокоточными значениями, полученные методом конфигурационного взаимодействия. При этом внимание акцентировано на ДМП между состояниями одинаковой симметрии. Ниже кратко рассмотрены уравнения метода ПЭП для возбужденных состояний и результаты расчетов ДМП как функция межъядерного расстояния для переходов: $A^{2} \Sigma^{+} \rightarrow X^{2} \Sigma^{+}, C^{2} \Sigma^{+} \rightarrow X^{2} \Sigma^{+}$и $D^{2} \Sigma^{+} \rightarrow X^{2} \Sigma^{+}$. Обсуждается также поведение параметров эффективного потенциала с изменением состояния.

\section{Уравнения метода ПЭП для возбужденных состояний}

Подходы, которые сочетают ТФП и метод оптимизированного эффективного потенциала, в принципе обеспечивают процедуру систематического улучшения точности расчетов. Уравнения, определяющие такой потенциал для основного состояния, могут быть найдены в различных источниках (см., например, $[16,18]$ ). Для определения возбужденных состояний, как правило, используется так называемая зависящая от времени ТФП. Один из методов расчета возбужденных состояний в рамках не зависящей от времени ТФП (см., например, [27-29]) был предложен в работах [20,21]. Ниже будут приведены только основные уравнения этого метода, которые использовались в численной имплементации (детали можно найти в [20,21]).

Известно, что в методе оптимизированного эффективного потенциала вариации орбиталей $\left\{\varphi_{i}\right\}$ должны удовлетворять одночастичному уравнению Шредингера с эффективным потенциалом $V_{\text {eff }}(\mathbf{r})$. Для основного состояния (нижний индекс „,“) они имеют вид

$$
\begin{gathered}
\left\{-\frac{1}{2} \nabla^{2}+V_{0 \mathrm{eff}}^{\sigma}(\mathbf{r})\right\} \varphi_{01}^{\sigma}(\mathbf{r})=\varepsilon_{0 i}^{\sigma} \varphi_{0 i}^{\sigma} \\
\sigma=\alpha, \beta, \quad i=1,2, \ldots, n .
\end{gathered}
$$

В случае, например, первого возбужденного состояния (ВС) на орбитали $\varphi_{j}^{\alpha}, j=1,2, \ldots, n^{\alpha}$, накладываются дополнительные условия

$$
\left\langle\varphi_{0 n}^{\alpha} \mid \varphi_{j}^{\alpha}\right\rangle=0
$$

которые обеспечивают ортогональность однодетерминантных волновых функций основного и возбужденного состояний, т. е. $\left\langle\Phi_{0} \mid \Phi\right\rangle=0$. Уравнения (4) означают, что все занятые ВС орбитали $\alpha$-роя ортогональны одной занятой орбитали $\varphi_{0 n}^{\alpha}$, соответствующей высшей орбитальной энергии $\varepsilon_{0 n}^{\sigma}$ в детерминанте $\Phi_{0}$.

В соответствии с методом асимптотического проектирования (АП) [20,21] указанная проблема сводится к следующим уравнениям для орбиталей первого ВС:

$$
\begin{gathered}
\left\{-\frac{1}{2} \nabla^{2}+V_{\mathrm{eff}}^{\alpha}(\mathbf{r})+\lambda_{0} P_{0 n}^{\alpha}\right\} \varphi_{i}^{\alpha}(\mathbf{r})=\varepsilon_{i}^{\alpha} \varphi_{i}^{\sigma}(\mathbf{r}), \\
\lambda_{0} \rightarrow \infty \\
\left\{-\frac{1}{2} \nabla^{2}+V_{\mathrm{eff}}^{\beta}(\mathbf{r})\right\} \varphi_{i}^{\beta}(\mathbf{r})=\varepsilon_{i}^{\beta} \varphi_{i}^{\beta}(\mathbf{r}),
\end{gathered}
$$

где $P_{0 n}^{\alpha}=\left|\varphi_{0 n}^{\alpha}\right\rangle\left\langle\varphi_{0 n}^{\alpha}\right|$. На практике значение $\lambda_{0}=10^{3}$ a.u. обеспечивало ортогональность $\left\langle\Phi_{0} \mid \Phi\right\rangle \sim 10^{-5}$ (см. также $[20,21])$.

Известно, что в расчетах орбитали представляются в виде разложения их по конечному числу базисных функций $\left\{c h i_{p}\right\}, p=1,2, \ldots, M$, т. е.

$$
\left.\left|\varphi_{i}^{\sigma}=\Sigma_{p=1}^{M} c_{p i}^{\sigma}\right| \chi_{p}\right\rangle, \quad \sigma=\alpha, \beta .
$$

Тогда уравнения (5) могут быть записаны в матричном виде, удобном для расчетов

$$
\Sigma_{q=1}^{M}\left(h_{\mathrm{mod}}^{\sigma}\right)_{p q} c_{q i}^{\sigma}=\varepsilon_{q i}^{\sigma} \Sigma_{q=1}^{M} S_{p q} c_{q i}^{\sigma}
$$

Для краткости здесь введены обозначения $S_{p q}=\left\langle\chi_{p} \mid \chi_{q}\right\rangle$ - матрица перекрывания и

$$
\begin{gathered}
h_{\mathrm{mod}}^{\alpha}=-1 / 2 \nabla^{2}+V_{e f f}^{\alpha}(\mathbf{r})+\lambda_{0} P_{0 n}^{\alpha}=h^{\alpha}+\lambda_{0} P_{0 n}^{\alpha}, \\
h_{\mathrm{mod}}^{\beta}=h^{\beta}=-\frac{1}{2} \nabla^{2}+V_{\mathrm{eff}}^{\beta}(\mathbf{r})
\end{gathered}
$$


и соответствующие матричные элементы

$$
\begin{gathered}
\left(h_{\text {mod }}^{\alpha}\right)_{p} q=\left\langle{ }_{p}\left|h^{\alpha}\right| \chi_{q}\right\rangle+\lambda_{0}\left\langle\chi_{p} \mid \varphi_{0 n}^{\alpha}\right\rangle\left\langle\varphi_{0 n}^{\alpha} \mid \chi_{q}\right\rangle, \\
\left(h_{\text {mod }}^{\beta}\right)_{p q}=\left\langle\chi_{p}\left|h^{\beta}\right| \chi_{q}\right\rangle .
\end{gathered}
$$

Следует отметить, что уравнения (7) не требуют вычисления двухэлектронных интегралов в отличие от уравнений метода ХФ. Более того, их решение не требует процедуры самосогласования.

После решения уравнений (7) полная энергия в однодетерминантном приближении определяется формулой

$$
\begin{aligned}
& E\{\varphi\}=\Sigma_{\sigma} \Sigma_{i}^{n^{\sigma}}\left\langle\varphi_{i}^{\sigma}\left|-\frac{1}{2} \nabla^{2}\right| \varphi_{i}^{\sigma}\right\rangle+\int \rho(\mathbf{r}) V_{\mathrm{ext}}(\mathbf{r}) d \mathbf{r} \\
& +\frac{1}{2} \int \rho(\mathbf{r}) V_{H}(\mathbf{r}) d \mathbf{r}+E_{x}\{\varphi\},
\end{aligned}
$$

где $\rho(\mathbf{r})$ - электронная плотность и $E_{x}\{\varphi\}-$ функционал обменной энергии, выраженной в терминах орбиталей уравнения (6):

$$
\begin{aligned}
E_{x}\{\varphi\} & =-\frac{1}{2} \Sigma_{\sigma} \Sigma_{i j}^{n^{\sigma}} \int d \mathbf{r} \int \frac{\varphi_{i}^{\sigma^{*}}(\mathbf{r})}{\left|\mathbf{r}-\mathbf{r}^{\prime}\right|} \varphi_{j}^{\sigma^{*}}\left(\mathbf{r}^{\prime}\right) \\
& \times \varphi_{i}^{\sigma}\left(\mathbf{r}^{\prime}\right) \varphi_{j}^{\sigma}(\mathbf{r}) d \mathbf{r}^{\prime}
\end{aligned}
$$

В заключение этого раздела следует отметить, что детерминант Слэтера, построенный из орбиталей уравнения (6), в общем случае не является собственной функцией оператора квадрата полного спина $\mathbf{S}^{2}$. В нашей реализации был использован один и тот же эффективный потенциал для $\alpha$ и $\beta$-роя, т.е. $V_{\mathrm{eff}}^{\alpha}(\mathbf{r})=V_{\mathrm{eff}}^{\beta}(\mathbf{r})=V_{\mathrm{eff}}(\mathbf{r})$. Такое упрощение ведет к генерации корректной по спину детерминантной функции.

\section{Дипольные моменты переходов и обсуждение результатов расчета}

Известно, что знание электронных дипольных моментов перехода составляет основу определения важных спектроскопических характеристик (например, [30-32]). Для системы из $N$ электронов соответствующие ДМП между $\Phi_{a}$ - и $\Phi_{b}$-состояниями могут быть определены в виде

$$
\mu_{a b}=\left\langle\Phi_{a}|\mu| \Phi_{b}\right\rangle
$$

где $\mu=e \sum_{k=1}^{N} \mathbf{r}_{k}-$ оператор дипольного момента c оператором координаты $\mathbf{r}_{k}$ для электрона с номером $k$. Это определение соответствует так называемому standard dipole-length approximation.

Альтернативно, если учесть коммутационные соотношения оператора координаты и гамильтониана, $\mu_{a b}$ может быть записан в виде

$$
\mu_{a b}=\frac{e \hbar^{2}}{\left(E_{b}-E_{a}\right) m} \sum_{k=1}^{N}\left\langle\Phi_{a}\left|\nabla_{k}\right| \Phi_{b}\right\rangle .
$$

Такое представление называют dipole velocity approximation. Здесь $m$ и $e-$ масса и заряд электрона.

С вычислительной точки зрения эти соотношения полезно переписать в атомных единицах:

$$
\begin{gathered}
\mu_{a b}=\sum_{k=1}^{N}\left\langle\Phi_{a}\left|r_{k}\right| \Phi_{b}\right\rangle, \\
\mu_{a b}=\frac{1}{E_{b}-E_{a}} \sum_{k=1}^{N}\left\langle\Phi_{a}\left|\nabla_{k}\right| \Phi_{b}\right\rangle .
\end{gathered}
$$

Конечно, указанные соотношения приводят к идентичным результатам для точных волновых функций $\Phi_{a}$ и $\Phi_{b}$. Однако для приближенных функций эти соотношения могут приводить к различным результатам. Наш вычислительный опыт с ХФ-функциями [24] показал, что определение (13) приводит к более корректным значениям $\mu_{a b}$, чем приближение dipole velocity approximation. Поэтому в данной работе мы использовали соотношения (13) для расчета ДМП. Соответствующие формулы для вычисления были взяты из монографии [33], где приведены выражения матричных элементов одночастичных операторов, построенные на разных детерминантах.

Используя орбитали из уравнения (7) и формулы (11)-(13), нами были рассчитаны энергии состояний $X^{2} \Sigma^{+}, A^{2} \Sigma^{+}, C^{2} \Sigma^{+}$и $D^{2} \Sigma^{+}$(табл. 1$)$, а также ДМП между состояниями одинаковой симметрии $A^{2} \Sigma^{+} \rightarrow X^{2} \Sigma^{+}$, $C^{2} \Sigma^{+} \rightarrow X^{2} \Sigma^{+}$и $D^{2} \Sigma^{+} \rightarrow X^{2} \Sigma^{+}$для разных межъядерных расстояний (табл. 2).

Все вычисления проводились в дистрибутивных базисах из $s$ гауссовых функций

$$
\begin{gathered}
\chi_{p}(x, y, z)=\exp \left\{-\zeta_{p}\left[x^{2}+y^{2}+\left(z-Z_{p}\right)^{2}\right]\right\}, \\
p=1,2, \ldots, M,
\end{gathered}
$$

параметры которых $\left\{\xi_{p}, Z_{p}\right\}$ определялись минимизацией энергии соответствующего состояния в однодетерминантном приближении, построенном на орбиталях уравнения (6). Другими словами, параметры базиса были подстроены под каждое индивидуальное состояние. Расчеты ДМП для НеН были проведены в базисах различной размерности $(12 s, 18 s$ и $24 s)$. Результаты показали, что базис из $18 s$ функций (15) представляет компромисс между точностью и затраченным компьютерным временем.

В табл. 1 сравниваются энергии состояний, рассчитанные методами ХФ и ПЭП для различных межъядерных расстояний. Как ожидалось, значения, полученные ПЭПметодом, несколько выше, чем энергии метода ХФ. При сравнении данных результатов со значениями энергий, полученными в работе [34] методом конфигурационного взаимодействия (КВ) с несколькими опорными конфигурациями, можно видеть, что средняя относительная погрешность (СОП) для интервала межъядерного расстояния $R=1.0-4.0 a_{0}$ (боровских радиусов, атомных единиц длины) сравнима для методов ХФ и ПЭП для различных состояний. Поведение параметров 
Таблица 1. Энергии основного и возбужденных состояний для разных межъядерных расстояний $R$, полученные в приближениях ХФ и ПЭП

\begin{tabular}{|c|c|c|c|c|c|c|c|c|}
\hline \multirow{2}{*}{$\begin{array}{l}R, a_{0} \\
(\mathrm{a} . \mathrm{u})\end{array}$} & \multicolumn{2}{|c|}{$X^{2} \Sigma^{+}$} & \multicolumn{2}{|c|}{$A^{2} \Sigma^{+}$} & \multicolumn{2}{|c|}{$C^{2} \Sigma^{+}$} & \multicolumn{2}{|c|}{$D^{2} \Sigma^{+}$} \\
\hline & $\mathrm{X} \Phi$ & ПЭП & $\mathrm{X} \Phi$ & ПЭП & ХФ & ПЭП & ХФ & ПЭП \\
\hline 1.0 & -3.055803 & -3.053287 & -3.008273 & -3.007516 & -2.933319 & -2.931105 & -2.922307 & -2.918015 \\
\hline 1.25 & -3.159281 & -3.157250 & -3.065457 & -3.063533 & -3.001299 & -2.9998377 & -2.982794 & -2.977218 \\
\hline 1.4 & -3.198745 & -3.196171 & -3.069518 & -3.068467 & -3.012724 & -3.012191 & -2.989650 & -2.984918 \\
\hline 1.5 & -3.219275 & -3.217968 & -3.066568 & -3.065869 & -3.014085 & -3.013661 & -2.988382 & -2.984586 \\
\hline 1.6 & -3.238485 & -3.234734 & -3.061050 & -3.060326 & -3.012570 & -3.010090 & -2.984526 & -2.980938 \\
\hline 1.75 & -3.261771 & 3.257059 & -3.052166 & -3.050348 & -3.009582 & -2.998694 & -2.975931 & -2.968971 \\
\hline 2.0 & -3.291615 & -3.284961 & -3.032034 & -3.029146 & -2.993941 & -2.986837 & -2.960183 & -2.957202 \\
\hline 2.5 & -3.328311 & -3.323285 & -3.006610 & -3.004801 & -2.975026 & -2.973199 & -2.936552 & -2.934972 \\
\hline 3.0 & -3.346388 & -3.342224 & -2.995195 & -2.993795 & -2.962964 & -2.961149 & -2.926103 & -2.925673 \\
\hline 3.5 & -3.354890 & -3.352067 & -2.990504 & -2.989055 & -2.957792 & -2.956179 & -2.922829 & -2.921390 \\
\hline 4.0 & -3.358725 & -3.357034 & -2.987985 & -2.987700 & -2.955979 & -2.953646 & -2.922013 & -2.919898 \\
\hline $\begin{array}{c}\text { СОП } \\
\%\end{array}$ & 1.3 & 1.4 & 1.4 & 1.5 & 1.3 & 1.5 & 1.3 & 1.4 \\
\hline
\end{tabular}

Примечание. СОП - средняя относительная погрешность в сравнении с методом конфигурационного взаимодействия ([34], таблицы 1-3).

Таблица 2. Сравнение методов ПЭП и ХФ: дипольные моменты перехода (а.и.) между состояниями $A^{2} \Sigma^{+} \rightarrow X^{2} \Sigma^{+}, C^{2} \Sigma^{+} \rightarrow X^{2} \Sigma^{+}$ и $D^{2} \Sigma^{+} \rightarrow X^{2} \Sigma^{+}$как функция межъядерного расстояния $R$

\begin{tabular}{c|c|c|c|c|c|c|c|c|c}
\hline \multirow{2}{*}{$R, a_{0}$} & \multicolumn{3}{|c|}{$A \rightarrow X$} & \multicolumn{3}{|c|}{$C \rightarrow X$} & \multicolumn{3}{c}{$D \rightarrow X$} \\
\cline { 2 - 9 } & ХФ & ПЭП & КВ [34] & ХФ & ПЭП & КВ [34] & ХФ & $\Pi Э \Pi$ & КВ [34] \\
\hline 1.0 & -0.1562 & -0.1686 & -0.1638 & -0.7461 & -0.8262 & -0.8447 & 0.2739 & 0.2829 & 0.2712 \\
1.25 & -0.5479 & -0.5927 & -0.6366 & -0.5202 & -0.5982 & -0.6084 & 0.2120 & 0.1998 & 0.2107 \\
1.4 & -0.5551 & -0.5877 & -0.6299 & -0.4309 & -0.4980 & -0.5077 & 0.2037 & 0.2064 & 0.2129 \\
1.5 & -0.5182 & -0.5703 & -0.5963 & -0.3973 & -0.4394 & -0.4583 & 0.2177 & 0.2102 & 0.2126 \\
1.6 & -0.4878 & -0.5193 & -0.5587 & -0.3471 & -0.3882 & -0.4200 & 0.2073 & 0.2194 & 0.2098 \\
1.75 & -0.4469 & -0.4617 & -0.5047 & -0.3105 & -0.3213 & -0.3781 & 0.1914 & 0.2303 & 0.2041 \\
2.0 & -0.3800 & -0.3745 & -0.4349 & -0.2817 & -0.3111 & -0.3357 & 0.1732 & 0.1917 & 0.1955 \\
2.5 & -0.3267 & -0.3259 & -0.3672 & -0.2351 & -0.2902 & -0.3069 & 0.1491 & 0.2210 & 0.2027 \\
3.0 & -0.3199 & -0.3163 & -0.3519 & -0.2686 & -0.3134 & -0.3251 & 0.1588 & 0.2300 & 0.2342 \\
3.5 & -0.3323 & -0.3377 & -0.3540 & -0.3179 & -0.3584 & -0.3785 & 0.2176 & 0.2636 & 0.2734 \\
4.0 & -0.3155 & 0.3459 & -0.3663 & -0.3514 & -0.4261 & -0.4495 & 0.2932 & 0.3198 & 0.3285 \\
\hline CОП\% \% & 11.2 & 7.8 & 0 & 15.1 & 5.7 & 0 & 5.6 & 3.3 & 0
\end{tabular}

Примечание. СОП - средняя относительная погрешность в сравнении с методом конфигурационного взаимодействия [34].

эффективного потенциала для возбужденных состояний с изменением межъядерного расстояния представлено на рис. $1-3$. Сплошная линия соответствует поведению параметра $C$, тогда как штриховая и пунктирная показывают зависимость параметров экспонент $d_{\mathrm{He}}$, и $d_{\mathrm{H}}$ соответственно. Как видно, тенденции в их поведении похожи для разных состояний. Например, параметры $C$ и $d_{\mathrm{He}}$, начиная с $R=1.25 a_{0}$, показывают очень слабую зависимость от межъядерного расстояния $R$, тогда как параметр экспоненты $d_{\mathrm{H}}$ имеет тенденцию уменьшаться при увеличении $R$. Это можно объяснить тем, что электронная плотность на атоме Н имеет более диффузный характер для больших $R$. Небольшие изменения в параметрах позволяют использовать их значения для
Таблица 3. Зависимость параметров эффективного потенциала от состояний при $R=1.5 a_{0}$ молекулы $\mathrm{HeH}$

\begin{tabular}{c|c|c|c|c}
\hline Параметры & $X^{2} \Sigma^{+}$ & $A^{2} \Sigma^{+}$ & $C^{2} \Sigma^{+}$ & $D^{2} \Sigma^{+}$ \\
\hline $\mathrm{C}$ & 1.015143 & 0.970657 & 1.212831 & 1.226026 \\
$d_{\mathrm{He}}$ & 1.538759 & 1.626953 & 1.306270 & 1.294525 \\
$d_{\mathrm{H}}$ & 1.214715 & 0.739604 & 0.609086 & 0.459572
\end{tabular}

данного $R$ как хорошее нулевое приближение для соседних точек потенциальной кривой. Подобное поведение экспоненциальных параметров $\left\{d_{\mathrm{He}}, d_{\mathrm{H}}\right\}$ с изменением возбужденных состояний можно наблюдать в табл. 3. 


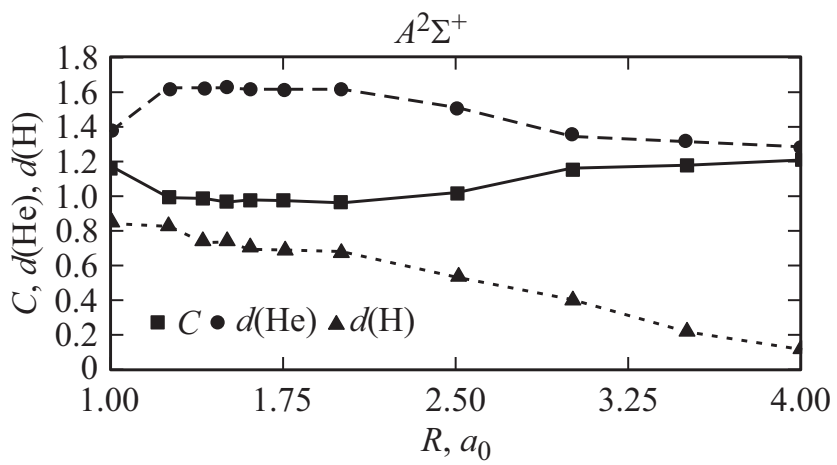

Pис. 1. Зависимость параметров эффективного потенциала от межъядерного расстояния $R$ для возбужденного состояния $A^{2} \Sigma^{+}$молекулы $\mathrm{HeH}$.

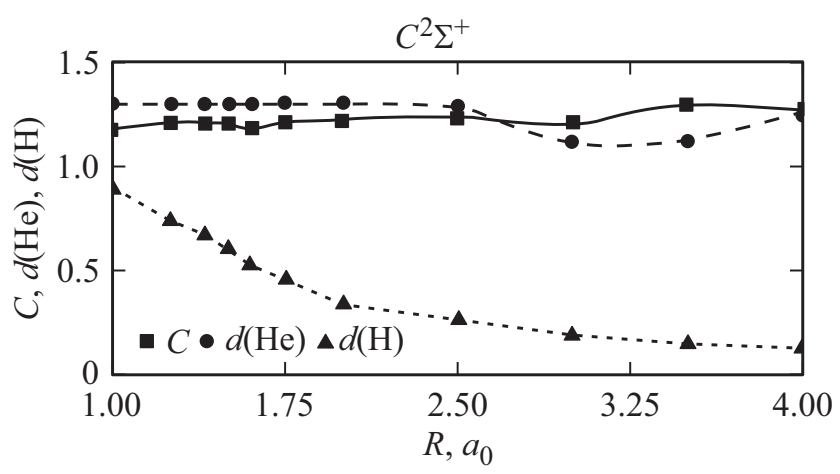

Рис. 2. Зависимость параметров эффективного потенциала от межъядерного расстояния $R$ для возбужденного состояния $C^{2} \Sigma^{+}$молекулы НеН.

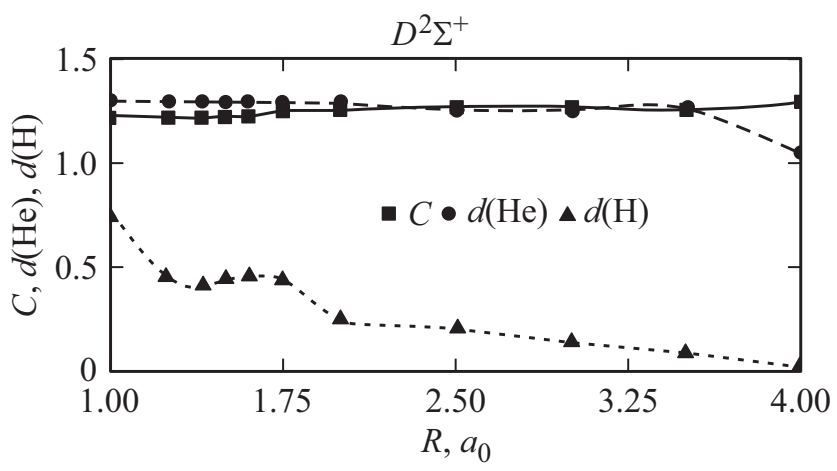

Pис. 3. Зависимость параметров эффективного потенциала от межъядерного расстояния $R$ для возбужденного состояния $D^{2} \Sigma^{+}$молекулы НеН.

Результаты расчета TDM, полученные ХФ-, ПЭПи КВ-методами, представлены в табл. 2. Результаты расчета показывают, что применение эффективного потенциала улучшает точность в определении ТДМ по сравнению с методом ХФ для всех рассмотренных состояний. При этом время расчета меньше, чем в методе ХФ, так как орбитали определяются одночастичным уравнением, решение которого не требует процедуры самосогласования. Наши результаты находятся в согласии с работой [35], где отмечалось, что локальные потенциалы, каким является потенциал (2), приводят к лучшим результатам для орбитальных энергий и других молекулярных свойств. Предложенный ПЭП-метод для расчета ДМП можно рассматривать как компромисс между вычислительными усилиями (по сравнению с КВ) и приемлемой точностью.

В заключение отметим, что эффективный потенциал в форме (2) допускает систематическое улучшение путем введения суммы экспоненциальных слагаемых, которые удовлетворяют условиям указанной ранее симметрии.

\section{Конфликт интересов}

Авторы заявляют, что у них нет конфликта интересов.

\section{Список литературы}

[1] Hohenberg P., Kohn W. // Phys. Rev. B. 1964. V. 864. P. 136.

[2] Kohn W., Sham L.J. // Phys. Rev. A. 1965. V. 1133. P. 140.

[3] Becke A.D. // J. Chem. Phys. A. 2014. V. 18. P. 140.

[4] Kryachko E.S., Ludena E.V. // Phys. Rep. 2014. V. 544. P. 123.

[5] Mardirossian N., Heard-Gordon M. // Mol. Phys. 2017. V. 115. P. 2315.

[6] Engel E., Dreizler R.M. // J. Comput. Chem. 1999. V. 20. P. 31.

[7] Cuevas-Saavedra R., Ayers P.W., Staroverov V.N. // J. Chem. Phys. 2015. V. 143. P. 244116.

[8] Fertig H.A., Kohn W. // Phys. Rev. A. 2000. V. 62. P. 52511.

[9] Weiner B., Trickey S.B. // Int. J. Quant. Chem. 1998. V. 69. P. 451.

[10] Görling A. // Phys. Rev. A. 1993. V. 47. P. 2783.

[11] Theophilou A.K. // J. Mol. Struct. (Theochem). 2010. V. 943. P. 42.

[12] Theophilou A.K. // J. Chem. Phys. 2018. V. 149. P. 074104.

[13] Nagy A. // J. Chem. Phys. 2018. V. 149. P. 204112.

[14] Theophilou A.K. // The Fundamentals of Electron Density, Density Matrix and Density Functional Theory in Atoms, Molecules and the Solid State / Ed. by Gidopoulos N.I., Wilson S. Dordrecht: Kluwer, 2003. P. 115.

[15] Theophilou A.K., Glushkov V.N. // J. Chem. Phys. 2006. V. 124. P. 034105.

[16] Sharp R.T., Horton G.K. // Phys. Rev. 1953. V. 90. P. 317.

[17] Slater J.C. // Phys. Rev. 1951. V. 81. P. 385.

[18] Talman J.D., Shadwick W.F. // Phys. Rev. A. 1976. V. 14. P. 36.

[19] Glushkov V.N. // Int. J. Quantum Chem. 2013. V. 113. P. 637.

[20] Glushkov V.N., Levy M. // J. Chem. Phys. 2007. V. 126. P. 174106.

[21] Glushkov V.N. // Opt. Spectrosc. 2005. V. 99. P. 684.

[22] Glushkov V.N., Wilson S. // Mol. Phys. 2012. V. 110. P. 149.

[23] Glushkov V.N., Fesenko S.I., Tsaune A.Ya. // Opt. Spectrosc. 2005. V. 98. P. 823.

[24] Glushkov V.N., Assfeld X. // J. Phys. B: At. Mol. Opt. Phys. 2017. V. 50. P. 125101.

[25] Glushkov V.N., Fesenko S.I. // Opt. Spectrosc. 2018. V. 124. P. 768.

[26] Henneker W.H., Popkie H.E. // J. Chem. Phys. 1971. V. 54. P. 1763. 
[27] Görling A. // Phys. Rev. A. 1996. V. 54. P. 3912.

[28] Levy M., Nagy A. // Phys. Rev. Lett. 1999. V. 83. P. 4361.

[29] Ayers P.W., Levy M., Nagy A. // Phys. Rev. A. 2012. V. 85. P. 042518.

[30] Devdariani A.Z. // J. Phys.: Conference Ser. 2019. V. 1289. P. 012018

[31] Tennyson J. // J. Mol. Spectrosc. 2014. P. 298. P. 1.

[32] Правилов А.М. Фотопроцессы в молекулярных газах. М.: Энергоатомиздат, 1992. 352 с.

[33] McWeeny R., Sutcliffe B.T. // Methods of Molecular Quantum Mechanics, Academic Press, London-New York, 1969. 380 p.

[34] Theodorakopoulos G., Petsalakis I.D., Nicolaides C.A., Buenker R.J. // J. Phys.: At. Mol. Phys. 1987. V. 20. P. 2339.

[35] Peach M.J.G., Kattirtzi J.A., Teale A.M., Tozer D.J. // J. Phys. Chem. A. 2010. V. 114. P. 7179. 